\title{
The running coupling in lattice Landau gauge with unquenched Wilson fermion and KS fermion
}

\section{Sadataka Furui* and Hideo Nakajima ${ }^{\dagger}$}

* School of Sci.\& Engr., Teikyo Univ., Utsunomiya, 320-8551 Japan

E-mail: furui@umb.teikyo-u.ac.jp

$\dagger$ Dept. of Infor. Sci., Utsunomiya Univ., Utsunomiya, 320-8585 Japan

E-mail: nakajima@is.utsunomiya-u.ac.jp

The running coupling of the Wilson fermon(JLQCD/CP-PACS) and that of Kogut-Susskind(KS) fermion(MILC) are measured in the lattice Landau gauge QCD in $\widehat{M O M}$ scheme. The quark propagator of the KS fermion is also measured and we find that it is infrared vanishing, which implies that quarks are confined. The renormalization factor of the running coupling and the tadpole renormalization define the scale of the quark wave function. Effects of the $A_{\mu}^{2}$ condensates of a few $\mathrm{GeV}^{2}$ are observed in the running coupling and also in the quark propagator.

XXIIIrd International Symposium on Lattice Field Theory

25-30 July 2005

Trinity College, Dublin, Ireland

${ }^{*}$ Speaker. 


\section{Introduction}

The mechanism of dynamical chiral symmetry breaking and confinement is one of the most fundamental problem of hadron physics. The propagator of dynamical quarks in the infrared region provides information on dynamical chiral symmetry breaking and confinement. In the previous paper[1], we measured gluon propagators and ghost propagators of unquenched gauge configurations obtained with quark actions of Wilson fermions (JLQCD/CP-PACS) and those of KogutSusskind(KS) fermions (MILC) $[2,3]$ in Landau gauge and observed that the configurations of the KS fermion are closer to the chiral limit than those of Wilson fermions.

In the analysis of running coupling obtained from the gluon propagator and the ghost propagator, with use of the operator product expansion of the Green function, we observed possible contribution of the quark condensates and $A^{2}$ condensates in the configurations of the KS fermion[1]. The quark propagator of quenched KS fermion was already measured in [4], and possible contribution of these condensates are reported. Unquenched KS fermion propagator of $20^{3} \times 64$ lattice $\left(\mathrm{MILC}_{c}\right)$ was measured in [5], but to distinguish the gluon condensates and the quark condensates, it is desirable to measure the quark propagator of larger lattice $\left(\mathrm{MILC}_{f}\right)$ and to compare with data of $\mathrm{MILC}_{c}$. We measure quark propagator of gauge configuration of 1) $\mathrm{MILC}_{c} 20^{3} \times 64, \beta=6.76$ and 6.83 and 2) MILC $_{f} 28^{3} \times 96, \beta=7.09$ and 7.11, using the Staple+Naik action[6].

\section{The running coupling in $\widetilde{M O M}$ scheme}

The running coupling in $\widetilde{M O M}$ scheme is given with use of the vertex renormalization factor $\tilde{Z}_{1}$ as

$$
\alpha_{s}(q)=\alpha_{R}\left(\mu^{2}\right) Z_{R}\left(q^{2}, \mu^{2}\right) G_{R}\left(q^{2}, \mu^{2}\right)^{2}=\frac{\alpha_{0}\left(\Lambda_{U V}\right)}{\tilde{Z}_{1}(\beta, \mu)^{2}} Z\left(q^{2}, \beta\right) G\left(q^{2}, \beta\right)^{2}
$$

where $Z$ and $G$ are the gluon and the ghost dressing function, respectively, and $\tilde{Z}_{1}$ is the vertex renormalization factor.

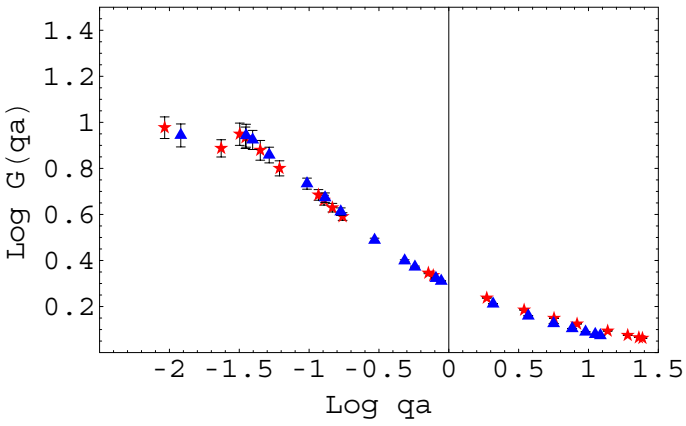

Figure 1: The $\log G(q a)$ as a function of $\log q a$ ( $a$ is the lattice spacing of MILC $_{f}$ ) of MILC $_{f} \beta_{\text {imp }}=7.09$ (stars) and MILC $_{c} \beta_{\text {imp }}=$ 6.76(triangles).

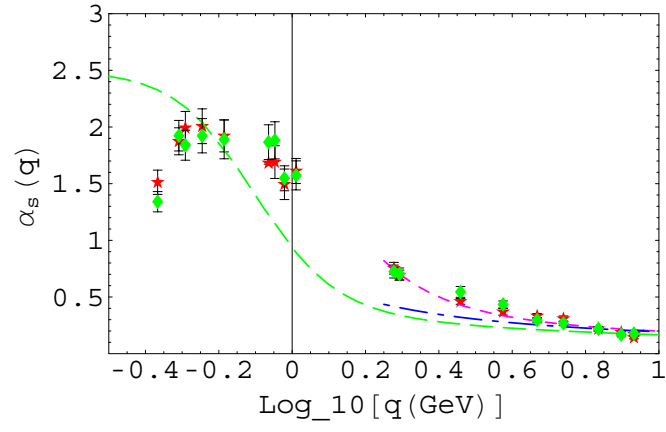

Figure 2: The running coupling $\alpha_{s}(q)$ as a function of $\log _{10} q(\mathrm{GeV})$ of $\operatorname{MILC}_{f} \beta_{\text {imp }}=$ 7.09(stars) and 7.11(diamonds). 
Our data suggests that the gluon propagator is infrared finite as in Dyson-Schwinger equation[9]. The running coupling in the infrared is suppressed as shown in Figure 2, but the main origin is the suppression of the ghost propagator in the infrared.

We parametrize the difference of the lattice data and the pQCD 4-loop result $[10,11]$ in the $1 \mathrm{GeV}<q<6 \mathrm{GeV}$ region in the form, with a minor correction term $d$ as

$$
\Delta \alpha_{s}(q)=\alpha_{s, l a t t}(q)-\alpha_{s, p e r t}(q)=\frac{c_{1}}{q^{2}}+\frac{c_{2}}{q^{4}}+d,
$$

where the $A^{2}$ condensates gives $c_{1}$ and the gluon condensates and/or quark condensates gives $c_{2}$. Although statistics is not large, running coupling of CP-PACS suggests $c_{1} \sim 2 \mathrm{GeV}$. The MILC data suggests $c_{1} \sim 4 \mathrm{GeV}$ and $c_{2} \sim-2 \mathrm{GeV}$. There is an analytical calculation that suggests correlation between the $A_{2}$ condensates and the the horizon function parameter[12].

\section{The quark wave function renormalization}

We renormalize the quark field as $\psi_{\text {bare }}=\sqrt{Z_{2}} \psi_{R}$, and define the colorless vector current vertex[7]

$$
\Gamma_{\mu}(q, p)=S^{-1}(q) G_{\mu}(q, p) S^{-1}(q+p)
$$

where

$$
G_{\mu}(p, q)=\int d^{4} x d^{4} y e^{i p \cdot y+i q \cdot x}\left\langle q(y) \bar{q}(x) \gamma_{\mu} q(x) \bar{q}(0)\right\rangle
$$

and $S(q)$ is the quark propagator.

The vertex of the vector current with $p=0$ is written as

$$
\Gamma_{\mu}(q)=\delta_{a, b}\left\{g_{1}\left(q^{2}\right) \gamma_{\mu}+i g_{2}\left(q^{2}\right) p_{\mu}+g_{3}\left(q^{2}\right) q_{\mu} \phi+i g_{4}\left(q^{2}\right)\left[\gamma_{\mu}, q\right]\right\}
$$

The Ward identity implies

$$
Z_{V}^{\widetilde{M O M}} \Gamma_{\mu}(q)=-i \frac{\partial}{\partial q^{\mu}} S^{-1}(q)
$$

where $\widetilde{Z_{V}^{M O M}} g_{1}\left(q^{2}\right)=Z_{\psi}\left(q^{2}\right)$ and $\widetilde{Z_{V}^{M O M}}=1$ when there is no lattice artefact.

The running coupling $g$ of the ghost, anti-ghost, gluon coupling

$$
g(q)=\tilde{Z}_{1}^{-1} Z_{3}^{1 / 2}\left(\mu^{2}, q^{2}\right) \tilde{Z}_{3}\left(\mu^{2}, q^{2}\right)
$$

and that of quark, gluon coupling

$$
g(q)=Z_{1}^{-1} Z_{3}^{1 / 2}\left(\mu^{2}, q^{2}\right) Z_{2}\left(\mu^{2}, q^{2}\right)
$$

are identical due to the Slavnov-Taylor identity. At the renormalization point $q=\mu$, we fix $Z_{2}\left(\mu^{2}, \mu^{2}\right)=1$ and $\tilde{Z}_{3}\left(\mu^{2}, \mu^{2}\right)=1$, and so $\tilde{Z}_{1}^{-1} Z_{3}^{1 / 2} \tilde{Z}_{3}=Z_{1}^{-1} Z_{3}^{1 / 2} Z_{2}$ implies $\tilde{Z}_{1}=Z_{1}$. On the other hand $Z_{\psi}\left(q^{2}\right)=\widetilde{Z_{V}^{M O M}} g_{1}\left(q^{2}\right)$ where for 16 tastes

$$
g_{1}\left(q^{2}\right)=\frac{1}{48 N_{c}} \operatorname{Tr}\left[\Gamma_{\mu}(q, p=0)\left(\gamma_{\mu}-q_{\mu} \frac{q}{q^{2}}\right)\right]
$$

When $\widetilde{Z_{V}^{M O M}}=1, g_{1}\left(\mu^{2}\right)$ is identical to $\tilde{Z}_{1}$ which is defined by the renormalization of the running coupling on the lattice defined at $\mu \sim 6 \mathrm{GeV}$ and summarized in Table 1 . 


\begin{tabular}{c|c|c|c|c} 
& $\beta_{1} / K_{\text {sea } 1}$ & $\beta_{2} / K_{\text {sea } 2}$ & average & configurations \\
\hline CP-PACS & $1.07(8)$ & $1.21(10)$ & 1.14 & $K_{\text {sea } 1,2}=0.1357,0.1382$ \\
\hline MILC $_{c}$ & $1.49(11)$ & $1.43(10)$ & 1.46 & $\beta_{1,2}=6.83,6.76$ \\
MILC $_{f}$ & $1.37(9)$ & $1.41(12)$ & 1.40 & $\beta_{1,2}=7.11,7.09$ \\
\hline
\end{tabular}

Table 1: The $1 / \tilde{Z}_{1}^{2}$ factor of the unquenched SU(3).

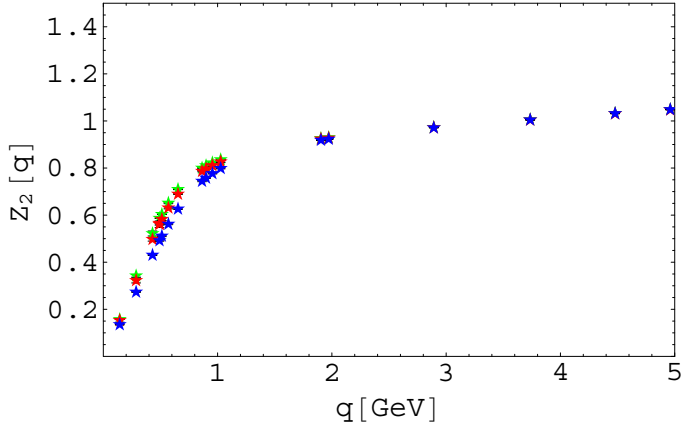

Figure 3: The quark propagator $Z_{2}(q)$ of MILC $_{f}$ with bear mass $m_{0}=13.6 \mathrm{MeV}$ (stars), 27.2MeV(diamonds) and 68.0MeV(triangles).

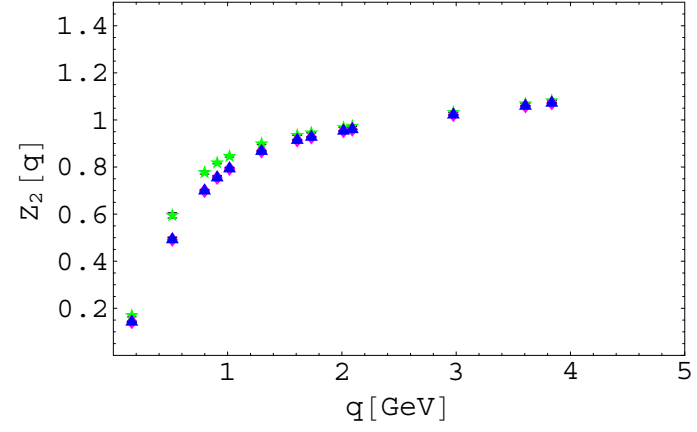

Figure 4: Same as Fig. 3 but quark of $\mathrm{MILC}_{c}$ with bear mass $m_{0}=11.5 \mathrm{MeV}$ (stars), 65.7MeV(diamonds) and $82.2 \mathrm{MeV}$ (triangles). The last two almost overlap.

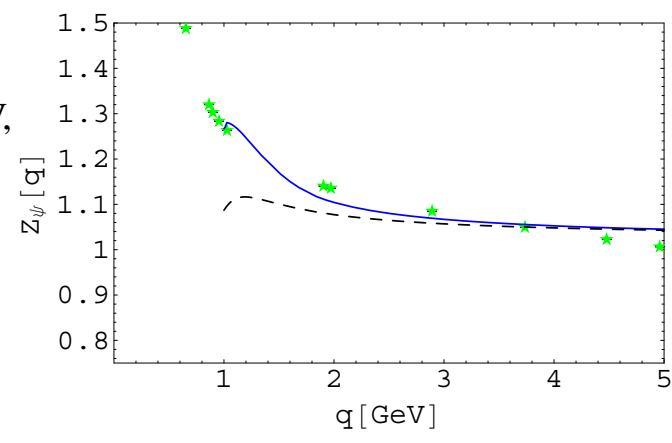

Figure 5: The quark inverse propagator $Z_{\psi}(q)$ of MILC $_{f}$ with bear mass $m_{0}=13.6 \mathrm{MeV}$. Dashed line is the pQCD result and solid line is the result including the $A_{\mu}^{2}$ condensates. in Figures 3 and 4. The apparent difference in the formulae of [5] and our work are only in the expression and in fact they are equivalent. However, [5] does not find this behavior of $Z_{2}(q)$.

Using the pQCD result of the inverse quark propagator[10, 11] and $\left\langle A^{2}\right\rangle$ and $\bar{c}_{2}$ (the contribution from the mixed condensate $\langle\bar{q} A q\rangle[8])$ as fitting parameters, we calculate

$$
Z_{\psi}(q)=\frac{1}{Z_{2}(q)}=Z_{\psi}^{\text {pert }}\left(q^{2}\right)+\frac{\left(\frac{\alpha(\mu)}{\alpha(q)}\right)^{\frac{-\gamma_{0}+\gamma_{A}{ }^{2}}{\beta_{0}}}}{q^{2}} \frac{\left\langle A^{2}(\mu)\right\rangle}{4\left(N_{c}^{2}-1\right)} Z_{\psi}^{\text {pert }}\left(\mu^{2}\right)+\frac{\bar{c}_{2}}{q^{4}}
$$

where $\alpha(q)$ are data calculated in the $\widetilde{M O M}$ scheme using the same $\operatorname{MILC}_{f}$ gauge configurations[1]. 
We estimate that $\bar{c}_{2}$ is small. In Figure 5, we show a fit of the MILC $_{f} m_{0}=13.6 \mathrm{MeV}$ data, by taking the renormalization point at $\mu \sim 3.8 \mathrm{GeV}$ with use of $\left\langle A^{2}(\mu)\right\rangle \sim 3.2 \mathrm{GeV}^{2}$ and $\bar{c}_{2}=0$. These parameters are consistent with [7].

In $q>1.5 \mathrm{GeV}$ region, dynamical mass of a quark in pQCD is expressed as[4]

$$
M(q)=-\frac{4 \pi^{2} d_{M}\langle\bar{q} q\rangle_{\mu}\left[\log \left(q^{2} / \Lambda_{Q C D}^{2}\right)\right]^{d_{M}-1}}{3 q^{2}\left[\log \left(\mu^{2} / \Lambda_{Q C D}^{2}\right)\right]^{d_{M}}}+\frac{m\left(\mu^{2}\right)\left[\log \left(\mu^{2} / \Lambda_{Q C D}^{2}\right)\right]^{d_{M}}}{\left[\log \left(q^{2} / \Lambda_{Q C D}^{2}\right)\right]^{d_{M}}},
$$

where $d_{M}=\frac{12}{33-2 N_{f}}$. The second term is the contribution of the massive quark. In the analysis of the lattice data, we observe that the quark condensates $-\langle\bar{q} q\rangle_{\mu}$ and $\Lambda_{Q C D}$ roughly satisfy $-\langle\bar{q} q\rangle_{\mu}=$ $\left(0.70 \Lambda_{Q C D}\right)^{3}[9]$, with $\Lambda_{Q C D}=0.69 \mathrm{GeV}$.

For the global fit of $M(q)$, we try the phenomenological monopole type[13]

$$
M(q)=\frac{c \Lambda^{3}}{q^{2}+\Lambda^{2}}+m_{0}
$$

where $m_{0}$ is the bare quark mass.

Figures 6 and 7 show the mass function $M(0)$ of $\mathrm{MILC}_{f}$ and $\mathrm{MILC}_{c}$, respectively.

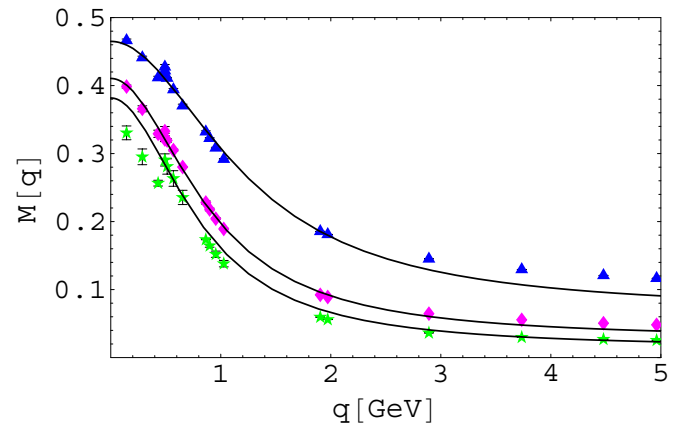

Figure 6: The dynamical mass of the $\mathrm{MILC}_{f}$ quark with bear mass $m_{0}=13.6 \mathrm{MeV}$ (stars), $27.2 \mathrm{MeV}$ (diamonds) and $68.0 \mathrm{MeV}$ (triangles) and the phenomenological fits.

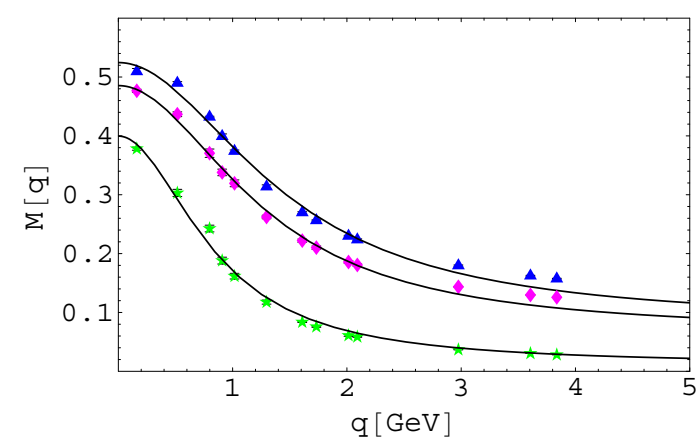

Figure 7: The dynamical mass of the $\mathrm{MILC}_{c}$ quark with bear mass $m_{0}=11.5 \mathrm{MeV}$ (stars), 65.7MeV(diamonds) and $82.2 \mathrm{MeV}$ (triangles) and the phenomenological fits.

We observe that the product $c \Lambda$ becomes larger as the bare quark mass becomes heavy and it depends on $\beta$ in the case of MILC $_{f}$ but not in the case of MILC $_{c}$. In the case of MILC $m_{0}=$ $13.6 \mathrm{MeV}$, the lowest three momentum points of $M(q)$ are systematically smaller than the other points. Ignoring these points we find $c \Lambda$ of $\beta=7.09$ is smaller than that of 7.11 and that of $\mathrm{MILC}_{c}$ as shown in Figure 8. In the chiral limit $m_{0} \rightarrow 0$, we obtain $M(0)=0.35 \sim 0.37 \mathrm{GeV}$, which is larger than [5] and that of the Wilson fermion[13] by about $20 \%$.

\section{Discussion and conclusion}

We measured running coupling of unquenched Wilson fermion and KS fermion and the quark wave function renormalization factor and mass function of the KS fermion. We observe that the quark propagator vanishes in the infrared, which implies that quark is confined. We fixed the scale of the quark propagator using the renormalization factor of the running coupling and that of the tadpole. 
A preliminary study of replacing the Staple+Naik action by the Asqtad action suggests that the $M(q)$ and $Z_{2}(q)$ of the two agree within $10 \%$.

\section{Acknowledgement}

We thank the MILC collaboration and JLQCD/ CP-PACS collaboration for supplying their gauge configurations in the data bases. We acknowledge discussion with Tony Williams, Christian Fischer and Patrick Bowman. H.N. is supported by the JSPS grant in aid of scientific research in priority area No.13135210. This work is supported by the KEK supercomputing project $05-$ 128.

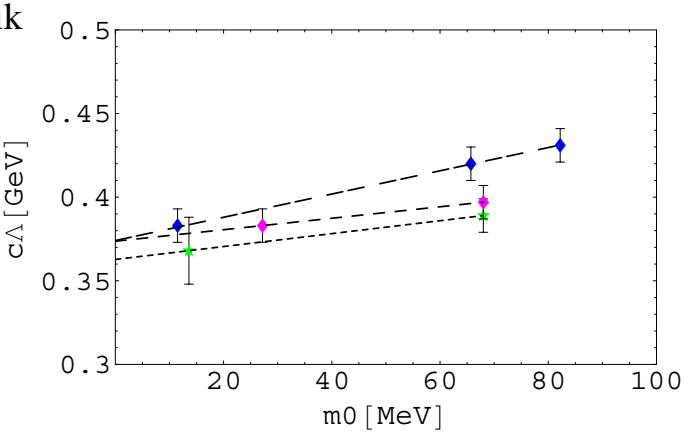

Figure 8: The mass function $c \Lambda$ as a function of bare mass and its chiral limit. Dotted line is the extrapolation of MILC $_{f} \beta=7.09$, dashed line is $\beta=7.11$ and the dash-dotted line is that of $\mathrm{MILC}_{c}$.

\section{References}

[1] S. Furui and H. Nakajima, Infrared features of the Kogut-Susskind fermion and the Wilson fermion in Lattice Landau Gauge QCD, hep-lat/0503029.

[2] C. Bernard et al., Quenched hadron spectroscopy with improved staggered quark action, Phys. Rev. D58(1998)014503.

[3] K. Orginos, D. Toussaint and R.L. Sugar, Variants of fattening and flavor symmetry restoration Phys. Rev. D 60(1999)054503.

[4] E.R. Arriola, P.O. Bowman and W. Broniowski, Landau-gauge condensates from quark propagator on the lattice, hep-ph/0408309.

[5] P.O. Bowman et al., Unquenched quark propagator in Landau gauge,Phys. Rev. D71(2005)054507, hep-lat/0402032.

[6] K. Orginos and D. Toussaint, Tests of Improved Kogut-Susskind Fermion Actions,Nucl. Phys. B(Proc. Suppl.)73(1999)909.

[7] Ph. Boucaud, F. de Soto, A. Le Yaouanc, J. Micheli, H. Moutarde, O Pène and J. Rodríguez-Quintero, Artefacts and $\left\langle A^{2}\right\rangle$ power corrections: revisiting the $M O M Z_{\psi}\left(p^{2}\right)$ and $Z_{V}$,hep-lat/0504017.

[8] M.J. Lavelle and M. Oleszczuk,Gauge-dependent condensates and the quark propagator, Phys. Lett. B275(1992)133.

[9] J.C.R. Bloch, Multiplicative renormalizability and quark propagator, Few Body Syst 33(2003)111.

[10] K.G. Chetyrkin and A. Rétay,Three-loop Three-Linear Vertices and Four-Loop $\widetilde{M O M} \beta$ functions in massless $Q C D$, hep-ph/0007088.

[11] K.G. Chetyrkin, Four-loop renormalization of QCD, hep-ph/0405193 v3(2005).

[12] D. Dudal, R.F. Soreiro, S.P. Sorella and H. Verschelde, The Gribov parameter and the dimension two gluon condensates in Eucledian Yang-Mills theories in the Landau gauge, hep-th/0502183.

[13] J.I. Skullerud and A.G. Williams, Quark propagator in the Landau gauge,Phys. Rev. D63(2001)054508. 